\title{
The identity of Brexit: A cultural psychology analysis
}

\author{
Giuseppe A. Veltri ${ }^{1}$ (D) | Rozlyn Redd ${ }^{2}$ | Terri Mannarini ${ }^{3}$ | \\ Sergio Salvatore ${ }^{3}$
}

${ }^{1}$ Department of Sociology and Social Research, University of Trento, Trento, Trentino Alto Adige, Italy

${ }^{2}$ Department of Infectious Disease Epidemiology, Imperial College London, UK

${ }^{3}$ Department of History, Society and Human Studies, University of Salento, Lecce, Puglia, Italy

\section{Correspondence}

Giuseppe A. Veltri, Department of Sociology and Social Research, University of Trento, Via Verdi 26, Trento 38122, IT, Italy.

Email: giuseppe.veltri@unitn.it

Funding information H2020 Societal Challenges, Grant/Award Number: 649436

\begin{abstract}
In this paper, we propose a culturalist interpretation of the outcome of the Brexit referendum. This interpretation is grounded on the analysis of the U.K.'s cultural milieu, which is part of a more general study of the cultural milieus of several European countries. Specific to the United Kingdom, our analysis is based on a questionnaire carried out on a representative sample of U.K. respondents stratified by sociodemographic variables and geographic region (NUTS1). Responses to the questionnaire were subjected to multidimensional analysis-a combination of multiple correspondence analysis and cluster analysis.

Findings about the multicountry analysis show how cultural milieus are characterized by five basic, embodied, affectladen, generalized worldviews (defined as "Symbolic Universes"). Four out of five symbolic universes consist of a way of interpreting reality by a specific anchorage (i.e., the ethical norm, the institutional order, the emotional experience interpersonal bond, and the belongingness). One symbolic universe can be viewed as the anomic reaction to the failure of those anchorages. Focusing on Brexit, by means of a discriminant analysis, it is showed how the incidence of the symbolic universes (and of the underpinning basic dimensions of meaning within the 12 U.K. macroregions') enables to identify differentially the macroregions with high proportion of "leave" at the Brexit referendum against those
\end{abstract}


with low proportion. More specifically, the high "leave" macroregion are characterized by higher relevance of cultural forms anchored on local identity/belongingness. Findings are discussed within the wider aims of the project and its implications for a cultural psychology informed policymaking.

\section{KEYWORDS}

Brexit, cultural psychology, identity, symbolic universes

\section{1 | INTRODUCTION}

On the 23rd of June 2016, the United Kingdom voted to leave the European Union after more than 40 years of membership and started a process of reducing a deep social and economic interdependence. Although polls indicated a substantial momentum of the "Leave campaign," the outcome came as a shock for a great many U.K. and European citizens. Since then, social scientists have started to study the reasons for this result and have investigated the anti-European Union (EU) sentiment that has taken root across several European countries. Analysing voting patterns during the referendum and sociodemographic variables, several studies focused on the role of people's education, income, and age in determining their choice to leave or remain in the EU. For example, Arnorsson and Zoega (2016) found that people were more likely to vote leave in regions where gross domestic product per capita is low, a high proportion of people have low education, and were over the age of 65 , there is strong net immigration, and people are more likely to be apprehensive about the EU. Another similar study focuses on age and education as the two main discriminants between voting remain and leave (Curtice, 2016). According to another study, Euroscepticism has become more widespread geographically and more narrow in terms of education levels (Goodwin \& Heath, 2016). A slightly different perspective is offered by Dorling (2016), who attributes much of the Leave vote Leave to anti-austerity policy reaction which was misattributed to the European level of policymaking.

To our knowledge, less attention has been paid to the role played by cultural factors in the referendum outcome. There are a few exceptions, such as the work of Henderson et al. (2016), which is however more a historical analysis of the relationship between English nationalism and Euroscepticism, and Kaufmann's (2016) analysis focuses on the role of value differences rather than sociodemographic variables in explaining the Brexit referendum. Following this line of research, the following paper proposes a cultural interpretation of the outcome of the Brexit referendum based on an empirical analysis of the role played by the U.K.'s cultural milieus on how people addressed the Remain/Leave choice.

Our interpretation is grounded on the Semiotic Cultural Psychology Theory (SCPT) framework and on the empirical basis provided by a more general and wider study of the cultural milieus of a comprehensive set of European countries (Cyprus, Denmark, Estonia, France, Germany, Greece, Italy, Malta, Netherlands, Spain, and the United Kingdom) carried out by the Re.Cri.Re. project-an international research programme funded by the Horizon 2020 "Societal challenges" call, involving 16 institutions of 12 countries of the European Union (www.recrire.eu; Ciavolino et al., 2017; Salvatore et al., 2018). The project, ended in May 2018, aimed at analysing the socio-cultural dynamics underpinning the political and institutional crisis affecting European societies. Re.Cri.Re. developed five research lines, and the present study is part of the third line (analysis of the role of cultural factors in shaping/affecting socio-political attitudes, choices, and behaviour). The Re.Cri.Re. project has made suggestions for designing "culture-oriented" policies aimed at addressing the crisis and rethinking Europe. 


\section{I THEORETICAL FRAMEWORK: SEMIOTIC CULTURAL PSYCHOLOGY THEORY}

In the last three decades, the rational choice model-that is, the mainstream way of interpreting socio-political and economic phenomena in terms of instrumental rationality-has been strongly criticized.

Several students-most of them having a sociological, anthropological, and/or psycho-social background-(e.g., Aronoff \& Kubik, 2013) have highlighted that the understanding of human behaviour and decision-making demands the comprehension of the cultural milieu of the community within-and in function of-which it is enacted.

Following this line of thought, the current study considers the cultural milieu as "...the social arena where people communicate, act, think and experience life and in so doing reproduce and elaborate [cultural meanings grounding and shaping sensemaking]" (Salvatore et al., 2018; p. 2). Accordingly, as intended here, the cultural milieu is the semiotic space consisting of the plurality of cultural meanings (i.e., symbolic universes and latent dimensions of sense, see below) mediating and shaping the forms of life that are active within a certain social group.

In order to analyse the cultural milieu and the role it plays in decision making-and, more specifically, in the Brexit referendum outcome-the current study adopts the SCPT as its theoretical and methodological framework.

SPCT has developed over the last two decades (Valsiner, 2007; Salvatore, 2016, Salvatore, 2018) within the more general domain of the socio-cultural psychology (Valsiner \& Rosa, 2007). It outlines a view of mental processes in terms of on-going dynamics of sensemaking, namely, processes of interpretation of the world through which persons try to make sense of their experience.

This processual view of culture as on-going processes of interpretation of the experience views people as acting to make their experience meaningful-that is, justifiable, thinkable, and reasonable-rather than as cognitively constrained elaborators of information.

Thus, making sense of experience is not only or mainly a cognitive task; it is an affirmation of belongingness to the social bond: Through feeling, thinking, and acting, people enact the generalized meaning that makes up their linkage to their social group. This does not mean that people are irrational; rather, it means that rationality, desire, and belongingness are reciprocally intertwined and constrained (Salvatore, 2018).

\section{1 | Sensemaking, symbolic universes, and cultural milieu}

Sensemaking is inherently social, embodied, and situated: People feel, think, and act not by following context-blind universal computational rules but by enacting sensemaking in terms of, and within the constraints of, generalized meanings embedded within the cultural milieu. Sensemaking works with basic intuitive embodied affect-laden assumptions concerning the world-what it is and how it works-as a whole (Ciavolino et al., 2017). SCPT adopts the term symbolic universes to denote such generalized meanings (Salvatore et al., 2018).

SCPT considers the cultural milieu to be heterogeneous: it consists of a plurality of symbolic universes, underpinning the immanent variability of the ways of feeling, thinking, and behaviour active within the social group. Each symbolic universe is an embodied, affect-laden interpretation of the same cultural environment (Salvatore, 2017)-where this interpretation consists in foregrounding some fundamental components of the experience-in the SCPT terms: latent dimension of sense; see below, following session) and backgrounding the others. This is the reason why those who are part of the same cultural milieu do not have the same feelings and do not think and act in the same way. Rather, the cultural milieu in which they partake-the symbolic universes comprising it-sets the domain, channelling and bounding the variability of their feelings, thoughts, and actions (for a similar view, see Carli \& Paniccia, 1999).

In short, SCPT conceives of the cultural milieu as the foundation of the variability of individual trajectories of sensemaking within a certain human community. Accordingly, cultural analysis is the way to understand not only and mainly what people share but also what makes them different from each other, in their visions of the world, therefore in their feelings, ideas, and actions. 


\subsection{The foci of the cultural analysis}

The view of the cultural milieu proposed above implies that its detection involves three main facets. First, the map of the main symbolic universes active within it: namely, the set of generalized meanings substantiating the worldviews, in terms of which members of the social group relate reciprocally and with the world.

Second, the cultural analysis is also aimed at understanding the structure grounding the symbolic universes, namely, the latent, basic, dimensions of sense underpinning them. Indeed, following the SCPT, each dimension of sense consists of the foregrounding of a basic component of experience (e.g., pleasantness and powerfulness; Venuleo, Salvatore, \& Mossi, 2015). Accordingly, latent dimensions of sense are the grounds for psychological life: Each dimension of sense foregrounds one component of the world, in so doing presentifies it as the content of experience (Salvatore, 2017). As said above, a symbolic universe can be viewed as consisting of one or more dimensions of sense made active (Salvatore, 2016).

It is worth adding that given their generalized affect-laden valence, a latent dimension of sense lends itself to being detected in terms of an oppositional structure-for example, pleasant versus unpleasant; trustworthy versus untrustworthy; familiar versus unfamiliar: The activation of the quality associated with one polarity is at the same time the neutralization of the opposite quality (for empirical analyses based on this method see Salvatore, Tonti, \& Gennaro, 2017; for a methodological discussion, see Salvatore \& Venuleo, 2013).

Third, the cultural analysis is interested to estimate the incidence of the symbolic universes within the social group partaking in the cultural milieu under investigation. According to this perspective, it is worth considering that any person can be profiled in terms of the symbolic universe being the most representative of her/his way of feeling/thinking/ acting. In so doing, on the one hand, the social group can be segmented in terms of the symbolic universes that are active within the cultural milieu embedding the social group; on the other hand, the differences in feeling, thoughts, and actions among segments can be explained in terms of the generalized meanings comprising each symbolic universe.

\section{3 | How to detect the cultural milieu}

Due to its embodied and super-individual, dynamic nature, the cultural milieu is not directly detectable. Its analysis requires an abductive modality of reasoning (Salvatore \& Venuleo, 2013), which reconstructs it from the analysis of the effects it exerts on the actors' modalities of sensemaking. In this case, the modalities of sensemaking being analysed are those triggered by the survey instrument (see Section 3.2 below).

The effect of a symbolic universe is shown by the fact that the person interprets her/his experience in terms of a network of meanings-opinions, claims, attitudes, feelings, and enactment-that cross over the plurality of domains and objects of experience of which her/his life consists (e.g., family life, job environment, view of the future, and system of values) in a sufficiently stable and homogeneous way, regardless of the semantic linkages among them. It follows that symbolic universes and the underpinning latent dimensions can be detected by means of a procedure of pattern recognition (implemented through multidimensional analysis, see Section 3 below) aimed at mapping the network of cooccurring meanings whose reciprocal association does not depend on their semantic linkages and therefore lends itself to be attributed to the generalizing effect of the basic cultural meanings-namely, the latent dimension of sense and symbolic universe's capacity to activate affective, presemantic linkages among the patterned signs (Salvatore et al., 2018).

\subsection{Aims and hypothesis}

This paper analyses if and how the U.K.'s cultural milieus have played a role in the Brexit referendum outcome. To this end, our work intends to study the association between the characteristics of the U.K.'s cultural milieus and the distribution of the Remain/Leave votes across the country. Our study tests the hypothesis that the U.K.'s macroregions with a higher proportion of Leave vote can be differentiated from those with lower proportion by reason of the differences of their cultural milieus (i.e., the different distribution of symbolic universes and latent dimension of sense among the macroregional territorial areas). 


\section{3 | METHOD}

The study consists of a secondary use of the part of the findings of the Re.Cri.Re. analysis that concern the U.K.'s cultural milieu. More specifically, the Re.Cri.Re. primary analysis provided an estimation of the cultural characteristics of each U.K. respondent included in the whole Re.Cri.Re. dataset (i.e., the symbolic universes representative of her/him and the level of association between him/her and the latent dimensions of sense, see Section 3.3). Secondary analysis was carried out based on these estimations.

Below, we provide a synthetic description of the method adopted for the primary analyses (details of the main Re.Cri.Re. analyses, henceforth Sample 1, can be found in Ciavolino et al., 2017, Salvatore et al., 2018, and Recrire consortium, 2017, at www.recrire.eu), whereas a more detailed picture of the methodological characteristics of the secondary analyses, specific of the current study, is provided (henceforth, Sample 2).

\section{1 | Samples construction}

Both Sample 1 and Sample 2 were extracted from the larger Re.Cre.Re. dataset collecting responses to the on-line Re.Cri.Re. survey. The dataset was collected from November 3, 2015 to June 6, 2016 (with the exception of respondents from the Netherlands and Denmark, collected during the Spring 2017).

Sample 1 is a homogeneous, nonproportional block sample $(N=727)$, randomly extracted from a larger dataset of subjects who had completed the on-line questionnaire, in accordance to an eight-block schema (gender*four levels of age $[<31 / 31-45 / 46-60 / />60])$, applied separately to each country; $n=9$ was the designed number of participants for each block. Countries were included in the analysis if the corresponding subsample presented at least five out of eight blocks with $n>5$. In so doing, 11 European countries were sampled (see above). Among these, six reached the designed distribution ( $n=9 * 8$ blocks)-Denmark, Estonia, Greece, Italy, Netherlands, and the United Kingdom. In other countries, most of blocks that could not be fully accomplished are those concerning the higher age level (>60 years).

Sample 1 was designed in accordance to the criterion of maximum variability, which states that the sample has to mirror as closely as possible the population's variability, regardless of the probability associated with states (Salvatore \& Venuleo, 2013). This is done with the aim of increasing the likelihood of including patterns of conditions/characteristics that even if quantitatively marginal may be theoretically and pragmatically relevant (e.g., a cultural niche triggering innovation). The three sample factors adopted for constructing Sample 1-country of residence, gender, and age-were chosen for the high likelihood of their being associated with cultural variability. Indeed, the country of residence is associated with institutional, linguistic, and territorial differences; both age and gender are allegedly associated with variable positions and practices within societies.

Sample 1's distribution between age levels is homogeneous for the first three levels $(27.5 \%, 27.1 \%, 26.2 \%$, respectively for $<31$ years, 31-45 years, 46-60 years); the oldest level is that with lower proportion (20.1\%). Needless to say, due to its nonproportional structure, Sample 1 is older than the European population (43.85 years $[S D=16,796$ vs. 41.47]). Gender is homogeneously distributed (women: $50.8 \%$ )-roughly the same distribution $(\mathrm{W}=51.2 \%)$ as in the European population. The distribution of education levels is over-represented in the higher levels-lower secondary or lower levels (less than 10 years of education): 19.3\%; upper secondary and postsecondary, nontertiary (10-13 years): $18.3 \%$; tertiary education (more than 13 years): $62.4 \%$.

Sample 2 was used for estimating the incidence of the latent dimensions of sense and symbolic universes within the U.K. cultural milieu as well as the sociodemographic profile of the symbolic universes within that country.

Sample 2 is a proportional quota random sample of the U.K. population $(N=765)$, stratified by NUTS1 regional encoding, gender and age (four levels-[<31/31-45/46-60//>60]; cf. Table 1; for further details, see Salvatore et al., 2018). Sample 2 proved $50.1 \%$ female; the average age was 44.98 ( $d s=16,187$ ). 
TABLE 1 Sample 2

\begin{tabular}{|c|c|c|c|c|c|c|c|c|c|c|}
\hline & Age/Sex & $<31 \mathrm{M}$ & $<31 \mathrm{~F}$ & $31-45 \mathrm{M}$ & $31-45 \mathrm{~F}$ & $45-60 \mathrm{M}$ & $45-60 \mathrm{~F}$ & $<60 \mathrm{M}$ & $<60 \mathrm{~F}$ & Tot \\
\hline East Midlands & $\begin{array}{l}N \\
\text { \% Samp. } \\
\text { \% Pop. }\end{array}$ & $\begin{array}{l}9 \\
1.17 \\
0.72\end{array}$ & $\begin{array}{l}8 \\
1.04 \\
0.69\end{array}$ & $\begin{array}{l}2 \\
0.26 \\
0.83\end{array}$ & $\begin{array}{l}11 \\
1.44 \\
0.85\end{array}$ & $\begin{array}{l}12 \\
1.57 \\
0.93\end{array}$ & $\begin{array}{l}9 \\
1.17 \\
0.95\end{array}$ & $\begin{array}{l}9 \\
1.17 \\
1.04\end{array}$ & $\begin{array}{l}3 \\
0.39 \\
1.19\end{array}$ & 63 \\
\hline East of England & $\begin{array}{l}\text { N } \\
\text { \% Samp. } \\
\text { \% Pop. }\end{array}$ & $\begin{array}{l}10 \\
1.31 \\
0.87\end{array}$ & $\begin{array}{l}5 \\
0.65 \\
0.83\end{array}$ & $\begin{array}{l}4 \\
0.52 \\
1.13\end{array}$ & $\begin{array}{l}12 \\
1.57 \\
1.16\end{array}$ & $\begin{array}{l}11 \\
1.44 \\
1.19\end{array}$ & $\begin{array}{l}12 \\
1.57 \\
1.22\end{array}$ & $\begin{array}{l}7 \\
0.91 \\
1.35\end{array}$ & $\begin{array}{l}5 \\
0.65 \\
1.57\end{array}$ & 66 \\
\hline Greater London & $\begin{array}{l}N \\
\text { \% Samp. } \\
\text { \% Pop. }\end{array}$ & $\begin{array}{l}7 \\
0.91 \\
1.57\end{array}$ & $\begin{array}{l}10 \\
1.31 \\
1.60\end{array}$ & $\begin{array}{l}10 \\
1.31 \\
2.19\end{array}$ & $\begin{array}{l}10 \\
1.31 \\
2.12\end{array}$ & $\begin{array}{l}9 \\
1.17 \\
1.48\end{array}$ & $\begin{array}{l}9 \\
1.17 \\
1.52\end{array}$ & $\begin{array}{l}10 \\
1.31 \\
1.18\end{array}$ & $\begin{array}{l}4 \\
0.52 \\
1.42\end{array}$ & 69 \\
\hline North East England & $\begin{array}{l}\text { N } \\
\text { \% Samp. } \\
\text { \% Pop. }\end{array}$ & $\begin{array}{l}3 \\
0.39 \\
0.42\end{array}$ & $\begin{array}{l}6 \\
0.78 \\
0.40\end{array}$ & $\begin{array}{l}7 \\
0.91 \\
0.45\end{array}$ & $\begin{array}{l}15 \\
1.96 \\
0.47\end{array}$ & $\begin{array}{l}10 \\
1.31 \\
0.53\end{array}$ & $\begin{array}{l}6 \\
0.78 \\
0.55\end{array}$ & $\begin{array}{l}9 \\
1.17 \\
0.59\end{array}$ & $\begin{array}{l}6 \\
0.78 \\
0.69\end{array}$ & 62 \\
\hline North West England & $\begin{array}{l}N \\
\text { \% Samp. } \\
\text { \% Pop. }\end{array}$ & $\begin{array}{l}6 \\
0.78 \\
1.12\end{array}$ & $\begin{array}{l}8 \\
1.04 \\
1.10\end{array}$ & $\begin{array}{l}10 \\
1.31 \\
1.31\end{array}$ & $\begin{array}{l}11 \\
1.44 \\
1.33\end{array}$ & $\begin{array}{l}8 \\
1.04 \\
1.41\end{array}$ & $\begin{array}{l}7 \\
0.91 \\
1.45\end{array}$ & $\begin{array}{l}6 \\
0.78 \\
1.53\end{array}$ & $\begin{array}{l}7 \\
0.91 \\
1.77\end{array}$ & 63 \\
\hline Northern Ireland & $\begin{array}{l}\text { N } \\
\text { \% Samp. } \\
\text { \% Pop. }\end{array}$ & $\begin{array}{l}7 \\
0.91 \\
0.29\end{array}$ & $\begin{array}{l}10 \\
1.31 \\
0.28\end{array}$ & $\begin{array}{l}6 \\
0.78 \\
0.35\end{array}$ & $\begin{array}{l}12 \\
1.57 \\
0.37\end{array}$ & $\begin{array}{l}11 \\
1.44 \\
0.36\end{array}$ & $\begin{array}{l}9 \\
1.17 \\
0.37\end{array}$ & $\begin{array}{l}5 \\
0.65 \\
0.34\end{array}$ & $\begin{array}{l}2 \\
0.26 \\
0.41\end{array}$ & 62 \\
\hline Scotland & $\begin{array}{l}N \\
\text { \% Samp. } \\
\text { \% Pop. }\end{array}$ & $\begin{array}{l}9 \\
1.17 \\
0.83\end{array}$ & $\begin{array}{l}5 \\
0.65 \\
0.83\end{array}$ & $\begin{array}{l}9 \\
1.17 \\
0.97\end{array}$ & $\begin{array}{l}9 \\
1.17 \\
1.02\end{array}$ & $\begin{array}{l}6 \\
0.78 \\
1.10\end{array}$ & $\begin{array}{l}12 \\
1.57 \\
1.16\end{array}$ & $\begin{array}{l}7 \\
0.91 \\
1.15\end{array}$ & $\begin{array}{l}6 \\
0.78 \\
1.39\end{array}$ & 63 \\
\hline South East England & $\begin{array}{l}\text { N } \\
\text { \% Samp. } \\
\text { \% Pop. }\end{array}$ & $\begin{array}{l}2 \\
0.26 \\
1.29\end{array}$ & $\begin{array}{l}11 \\
1.44 \\
1.24\end{array}$ & $\begin{array}{l}10 \\
1.31 \\
1.66\end{array}$ & $\begin{array}{l}10 \\
1.31 \\
1.72\end{array}$ & $\begin{array}{l}11 \\
1.44 \\
1.78\end{array}$ & $\begin{array}{l}7 \\
0.91 \\
1.81\end{array}$ & $\begin{array}{l}9 \\
1.17 \\
1.93\end{array}$ & $\begin{array}{l}3 \\
0.39 \\
2.27\end{array}$ & 63 \\
\hline South West England & $\begin{array}{l}N \\
\% \text { Sa } \\
\% \text { Pop. }\end{array}$ & $\begin{array}{l}6 \\
0.78 \\
0.80\end{array}$ & $\begin{array}{l}10 \\
1.31 \\
0.75\end{array}$ & $\begin{array}{l}10 \\
1.31 \\
0.93\end{array}$ & $\begin{array}{l}7 \\
0.91 \\
0.95\end{array}$ & $\begin{array}{l}7 \\
0.91 \\
1.08\end{array}$ & $\begin{array}{l}10 \\
1.31 \\
1.11\end{array}$ & $\begin{array}{l}10 \\
1.31 \\
1.34\end{array}$ & $\begin{array}{l}3 \\
0.39 \\
1.57\end{array}$ & 63 \\
\hline Wales & $\begin{array}{l}\text { N } \\
\text { \% Samp. } \\
\text { \% Pop. }\end{array}$ & $\begin{array}{l}2 \\
0.26 \\
0.49\end{array}$ & $\begin{array}{l}10 \\
1.31 \\
0.47\end{array}$ & $\begin{array}{l}5 \\
0.65 \\
0.53\end{array}$ & $\begin{array}{l}14 \\
1.83 \\
0.54\end{array}$ & $\begin{array}{l}12 \\
1.57 \\
0.60\end{array}$ & $\begin{array}{l}6 \\
0.78 \\
0.63\end{array}$ & $\begin{array}{l}11 \\
1.44 \\
0.73\end{array}$ & $\begin{array}{l}2 \\
0.26 \\
0.85\end{array}$ & 62 \\
\hline West Midlands & $\begin{array}{l}N \\
\text { \% Samp. } \\
\text { \% Pop. }\end{array}$ & $\begin{array}{l}6 \\
0.78 \\
0.91\end{array}$ & $\begin{array}{l}10 \\
1.31 \\
0.88\end{array}$ & $\begin{array}{l}9 \\
1.17 \\
1.05\end{array}$ & $\begin{array}{l}11 \\
1.44 \\
1.06\end{array}$ & $\begin{array}{l}11 \\
1.44 \\
1.10\end{array}$ & $\begin{array}{l}5 \\
0.65 \\
1.12\end{array}$ & $\begin{array}{l}8 \\
1.04 \\
1.22\end{array}$ & $\begin{array}{l}7 \\
0.91 \\
1.42\end{array}$ & 67 \\
\hline Yorkshire and the Humber & $\begin{array}{l}N \\
\text { \% Samp. } \\
\text { \% Pop. }\end{array}$ & $\begin{array}{l}9 \\
1.17 \\
0.88\end{array}$ & $\begin{array}{l}11 \\
1.44 \\
0.85\end{array}$ & $\begin{array}{l}5 \\
0.65 \\
0.98\end{array}$ & $\begin{array}{l}8 \\
1.04 \\
0.99\end{array}$ & $\begin{array}{l}11 \\
1.44 \\
1.05\end{array}$ & $\begin{array}{l}8 \\
1.04 \\
1.07\end{array}$ & $\begin{array}{l}8 \\
1.04 \\
1.14\end{array}$ & $\begin{array}{l}3 \\
0.39 \\
1.33\end{array}$ & 63 \\
\hline Tot & & & & & & & & & & 766 \\
\hline
\end{tabular}

\section{2 | Instrument}

The analysis of symbolic universes is based on the VOC questionnaire (View of Context). VOC is a 68-item questionnaire that maps how people represent affect-laden, significant aspects of their life and context. Previous studies conducted on Italian and English versions of the questionnaire have shown a satisfactory construct validity of the questionnaire (Carli \& Salvatore, 2001; Ciavolino et al., 2017; Mannarini, Nitti, Ciavolino, \& Salvatore, 2012) as well as a satisfactory level of inner consistency (Alfa value above 0.70, cf. Ciavolino et al., 2017 and Supporting Information).

The questionnaire's items were constructed on the grounds of a methodology integrating psychoanalytic and psycho-cultural standpoints (Carli \& Paniccia, 1999; Mannarini et al., 2012; Osgood, Suci, \& Tannenbaum, 1957; Salvatore \& Venuleo, 2013) aimed at detecting the oppositional structures underpinning the modes of interpreting reality. According to this methodology, workable items facilitate the expression of perceptions/opinions/judgments concerning the microsocial and macrosocial environment (e.g., evaluation of the place where the person lives, level of trustworthiness of social structures) and social identity (e.g., moral judgments on critical social behaviours) and 
in so doing to trigger the activation of generalized meanings (for a discussion, see Ciavolino et al., 2017). Details on the VOC are reported in Salvatore, Fini, Mannarini, Valsiner, and Veltri (forthcoming) and Supporting Information.

\section{3 | Data analysis}

The procedure of pattern recognition aimed at detecting the latent dimensions of sense and the symbolic universes was carried out by means of a multidimensional procedure of analysis, combining multidimensional correspondence analysis and cluster analysis (CA). We carried out the following steps:

1. First, the Sample 1 data set underwent multiple correspondence analysis (MCA). MCA is a method for mapping relations that are active within the dataset in a parsimonious way. These relations are summed up in terms of a limited number factorial dimensions being able to map the greatest amount of the information contained initially in the dataset (Benzecri, 1992). Each factorial dimension extracted by the MCA describes the juxtaposition between two patterns of co-occurring response modalities across respondents. According to the methodological framework adopted, and consistently with other authors (see, for example, Landauer, Foltz, \& Laham, 1998), each factorial dimension is viewed as the marker of a latent dimension of sense.

2. After applying MCA, CA-hierarchical classification method-was carried out, in order to identify the response profiles that were active in the dataset, each one associated with a different group of individuals. The identification of profiles was based on the criterion of maintaining the maximum similarity between the response profiles grouped in the same cluster and identifying the maximum difference in the response profiles among different clusters. In our case, the similarity/dissimilarity criterion is given by the main factorial dimensions extracted by the previous MCA.

3. Each cluster produced by the CA consists of a specific profile of individual responses, namely, a pattern of responses that tends to co-occur redundantly over the sample. Thus, each cluster was interpreted as the marker of a symbolic universe, associated with a certain group of respondents characterized by that response profile.

The multidimensional correspondence analysis provides an index of association (factorial coordinate) between each individual in the analysis and the factorial dimensions extracted. Moreover, CA provides a classification of individuals in the cluster most similar to the identified response profile.

These indexes were then calculated for Sample 2 respondents. Technically speaking, this was done by introducing the Sample 2 respondents in the MCA-CA performed on Sample 1, yet as illustrative individuals (i.e., cases that do not contribute to the estimation of the factorial dimensions and clusters but are described by them, once defined). In so doing, the measurements of the parameters produced by the Re.Cri.Re. primary analysis based on Sample 1 were used to estimate the cultural profile of the Sample 2 respondents, namely, the degree of association with the latent dimensions of sense and the symbolic universes in terms of which each of them can be characterized.

Thus, the main MCA factorial dimensions and the cluster membership was obtained for each participant of Sample 2. On this basis, the incidence of the latent dimension of sense and symbolic universes within each U.K. macroregional area (NUTS1) were computed.

In order to test our hypothesis of the association between the macroregion cultural milieus and the Brexit referendum outcome, a discriminant analysis was performed (stepwise method). Discriminant analysis is a procedure aimed at identifying the more parsimonious set of variables (predictors) whose combination is able to account for the differences among groups of cases, grouped previously by a classificatory variable. Discriminant analysis provides three main results: (a) the classificatory function, namely, the model that maps the differences among groups of cases in terms of the criterion variables; (b) the estimation of the discriminative capacity of the function (Wilks' Lambda is the parameter used for this purpose) as well as its statistical significance (based chi square metric); (c) the estimation of the efficacy of the model, in terms of the comparisons between the original classification of cases and that 
performed by the discriminant function. In order to carry out the discriminant analysis, the 12 NUTS1 territorial sites of the United Kingdom were grouped into two classes, in accordance with the percentage of Leave votes out of the whole electoral population (i.e., encompassing both nonvalid votes and people not voting) in each macroregion (data were retrieved from the U.K. electoral commission; www.electoralcommission.org.uk). The median was used as cut-off, thus distinguishing the regional areas with more than $39 \%$ and with less than $40 \%$ of Leave votes out of the whole electorate (cf. Table 2).

A set of indicators concerning the characteristics of the cultural milieu were used and predictive variables. More particularly, 12 indicators were defined:

- Six indicators concerning the incidence of both positive and negative polarities of the three main factorial dimensions (to this end, the highest and lowest of whole Sample 1's quartile was used as threshold, respectively, for positive and negative polarity).

- Five indicators concerned the incidence of the clusters extracted by the CA.

- One indicator concerned the variability of the symbolic universes within the macroregion's cultural milieu. It was calculated as the difference between the empirical frequency of symbolic universes in the macroregional area and the condition of their equal distribution (the difference was calculated in accordance to the chi-square metric, namely, as square root of the sum of the square difference).

Indicators were applied separately on the 12 U.K. NUTS1 macroregions. The last step consisted in finding the discriminative function with the cultural indicators so defined used as predictive variables.

\section{4 | RESULTS}

\section{1 | Latent dimensions of sense}

The MCA extracted three main factorial dimensions (for a full description see Salvatore et al., forthcoming). Below, we present a synthetic interpretation of the dimensions, based on the analysis of the set of co-occurring items associated with each polarity of the factorial dimension. Needless to say, other interpretations could be possible, but these had a satisfying level of interrater reliability (see Salvatore et al., forthcoming). These factorial dimensions

TABLE 2 NUTS1 U.K. classification in accordance to Brexit referendum results

\begin{tabular}{lll|}
\hline NUTS1 region & Leave/electorate & LEAVE versus ELECT \\
\hline East England & 42.75 & 2 \\
\hline East Midlands & 43.60 & 2 \\
\hline London & 27.89 & 1 \\
\hline North-east England & 40.23 & 2 \\
\hline North-west England & 37.53 & 1 \\
\hline Northern Ireland & 27.71 & 1 \\
\hline Scotland & 25.54 & 1 \\
\hline South-east England & 39.72 & 1 \\
\hline South-west England & 40.35 & 2 \\
\hline Wales & 37.64 & 1 \\
\hline West Midlands & 42.65 & 2 \\
\hline Yorkshire and The Humber & 40.77 & 2 \\
\hline
\end{tabular}

Note. 1 = Leave $<40 ; 2$ = Leave $>39 \%$. 
correspond to $35.4 \%$ of the whole inertia (respectively, 19.9\%, 10.9\%, 4.6\%; the inertia associated with each factor was calculated in accordance with the Benzecri's simplified formula of revaluation). This is a reasonably large portion of variance when one considers that it is the output of a MCA, which distributed the variability over very many factorial dimensions.

\subsubsection{Affective connotation of the world-Friend versus foe}

The first factorial dimension polarizes two opposite generalized, affect-laden ways of connoting the field of experience as a whole. On the one hand, a positive connotation that qualifies the world as a fine, trustworthy object, lending itself to be engaged with; on the other hand, a negative connotation qualifying it as unfair, meaningless, and unreliable. We therefore interpret the factorial dimension as the marker of the latent dimension of sense consisting of the very basic, affective connotation of the world, in terms of the generalized opposition: foe/friend.

\subsection{2 | Direction of desire-Passivity versus engagement}

According to the interpretation of the polarities provided above, the second factorial dimension can be viewed as the marker of the latent dimension of sense consisting of the direction of desire, namely, the position assumed with regard the world: passivity versus engagement. Passivity is characterized by the sense of dependency on institutions, agencies, and primary network, thanks to which the subject can cope with an uncertain world; Engagement is characterized by the sense of agency, fostered by trust in people and institutions. In the final analysis, this latent dimension of sense concerns the meaning of the world as the source of action directed towards the subject (i.e., passivity) or, in contrast, as the goal of the subject's investment (i.e., engagement). In other words, being the object or the subject of desire (investment, commitment, action on).

\subsubsection{Form of demand-Demand for systemic resources versus demand for community identity}

In sum, the polarities characterizing this factorial dimension can be interpreted as the marker of the latent dimension of sense consisting of the opposition between what we propose to consider as two forms of demand, namely, two basic views of one's fundamental need: the demand for systemic resources versus the demand for community bond. In the former case, the demand concerns functional devices and services one needs in order to address a challenging, uncertain world; in the latter case, the demand concerns the need to make life meaningful in spite of the untrustworthiness of institutions, where the meaning lies in the significant, vital participation in community bonds, namely, bonds that go beyond the primary linkages (i.e., beyond the relation within family and close friends).

\section{2 | Symbolic universes}

The partition in five clusters was chosen as the optimal cluster analytic solution (interclass inertia/total inertia: $0.1910 / 0.4588=0.4163$ ). Further differentiation would not have greatly increased the interclass/total inertia ratio (e.g., ratio corresponding to six clusters: 0.4531 ), or have improved the meaningfulness of the partition. Profiles of responses obtained by the $\mathrm{CA}$ as well as their interpretation in terms of symbolic universes are provided by Salvatore et al. (2018). Below, we present a synthetic summary of this more complete interpretation.

\subsection{1 | Symbolic universe 1: Ordered universe}

This worldview is characterized by two important facets. On the one hand, a generalized positive attitude towards the world (institutions and services, the people, the place where one lives, the country, the future) which is considered trustworthy, receptive of the efforts to engage with and to improve it. On the other hand, there is identification with transcendent values and ideals (e.g., justice, morality, solidarity; rejection of opportunism, conformism, and 
power) that foster commitment to making things better-where such commitment is seen as a value in itself: the way of making life meaningful, rather than of pursing material interests. The combination of these two facets outlines what we interpret as the basic assumption substantiating this symbolic universe: faith in the inherent ethical order of the world. Rightness, morality, and efficacy go together, what is just is also efficacious in rendering things better, because the universe follows its own harmonious design. Behaviour has to conform to and reflect this universal order and in so doing one can trust in being on the right side of history.

\subsection{2 | Symbolic universe 2: Interpersonal bond}

This symbolic universe comprises a group of responses detecting a positive, optimistic vision of the world, as a place that is meaningful and fulfilling. On the other hand, the world these responses refer to is not the universalistic one of the previous symbolic universe; rather, it is the vital world of interpersonal, emotional bonds. To be part of such a world is an end in itself: Sacrifices (in terms of adaptability and conformism) are needed for it and are repaid in terms of safety and fulfilment, as well as in promoting a moderate sense of agency, trust, and openness to the new. The line of the famous song-all you need is love-depicts the basic assumption of this symbolic universe.

\subsection{3 | Symbolic universe 3: Caring society}

This symbolic universe is characterized by a vision of society and institutions as trustworthy providers of services and commons (e.g., education, health, security, and development). Society is receptive to the demands and needs of people. This vision fosters a generalized feeling of confidence in life, optimism in the future and a sense of agency -What one has to do is to keep oneself within the rules of the game, there being those who take care of handling it for the best. It is worth noting how in the case of this symbolic universe, the trustworthiness attributed to institutions does not mean passivity and dependency; rather, it works as grounds for a sense of agency: People who identify with this symbolic universe feel able to pursue purposes because they feel part of a system that supports and allows their efforts.

\subsection{4 | Symbolic universe 4: Niche of belongingness}

This symbolic universe shares a similar anchorage to the primary network characterizing Interpersonal bond. Yet, in this case, such an anchorage is combined with a negative generalized connotation of the world outside the primary network-in terms of pessimism about the future, fatalism, untrustworthiness of agencies and institutions. In such a context, the primary network is a necessity responding to the need of finding shelter from and surviving the anomic, threatening outside, rather than a matter of pleasure, an end in itself. Consistent with such a feeling, the primary network is connoted in terms of family-centred power.

\subsection{5 | Symbolic universe 5: Others' world}

This symbolic universe outlines a fully negative, even desperate vision of the world-generalized untrustworthiness, sense of impotency, lack of agency, anomie. The world belongs to those who have power-the defeated have only the chance to try to survive day-by-day, surrendering to those with the power to lead the game. Morality and values are a luxury one cannot afford when the only possible concern is to limit the damage.

\subsection{The incidence of symbolic universes within the U.K.'s cultural milieu}

Figure 1 reports the distribution of the symbolic universes within the U.K. sample (i.e., Sample 2). Niche of belongingness shows the highest incidence, whereas Caring society shows the lowest.

The 12 NUTS1 macroregions proved not to differ with each other as to the distribution of the symbolic universes within them (chi-square $=46,379[d f=44], p=0.374)$. 


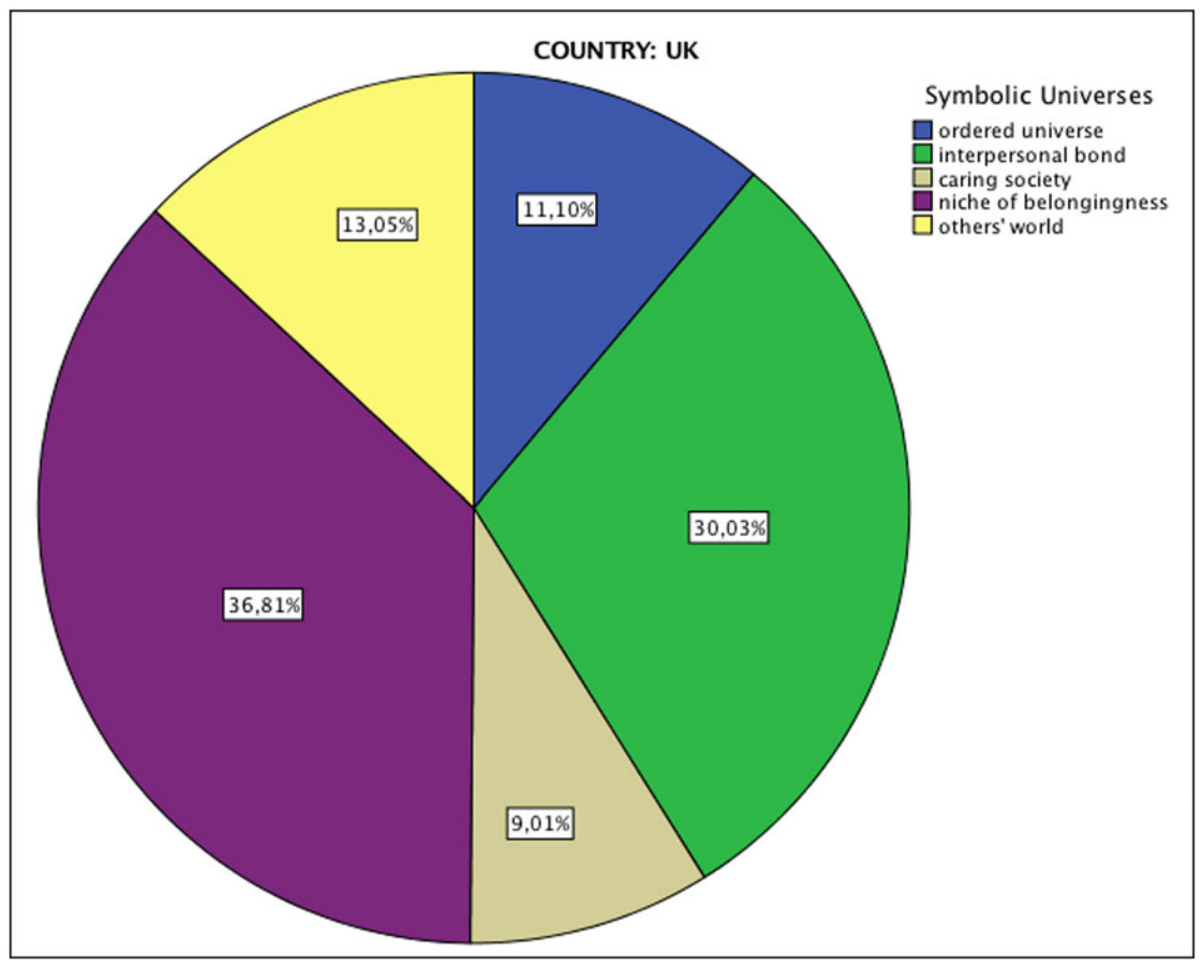

FIGURE 1 Incidence of the symbolic universes in U.K.'s cultural milieus [Colour figure can be viewed at wileyonlinelibrary.com]

\subsection{Cultural milieu and Brexit referendum outcome}

The discriminant analyses (stepwise method) produced a significant function having $100 \%$ classification success rate. (Wilks' Lambda 0.006, canonical correlation: 0.997; chi-square = 25.707 [ $d f=10], p<0.004$ ).

Table 3 reports the second-ordered cultural indicator included in the standardized canonical discriminant functions. On the basis of the group centroids (Table 4), one can see how the regional sites with a proportion of Leave equal to or higher than $40 \%$ are discriminated by the combination that is characterized mainly by high incidence of the following three symbolic universes: interpersonal bond, niche of belongingness and ordered universe and low level of cultural heterogeneity, incidence of people with attitudes of engagement and connoting the world in terms of foe.

TABLE 3 Discriminant analysis

\begin{tabular}{|rr}
\hline Second-order cultural indicators & Function \\
\hline Incidence symbolic universe: ordered universe & 21,807 \\
\hline Incidence of symbolic universe: interpersonal bond & 30,292 \\
\hline Incidence of symbolic universe: caring society & 9,42 \\
\hline Incidence of symbolic universe: niche of belongingness & 22,224 \\
\hline Variability of symbolic universes & $-12,542$ \\
\hline Affective connotation of the world: Friend & $-4,124$ \\
\hline Affective connotation of the world: Foe & $-10,229$ \\
\hline Direction of desire: Passivity & 2,641 \\
\hline Direction of desire: Engagement & $-12,892$ \\
\hline Demand for Systemic resources & 3,814 \\
\hline
\end{tabular}

Note. Standardized coefficient of the canonical discriminant function. 
TABLE 4 Discriminant analysis: Group centroids of two classification options for each NUTS1 region

\begin{tabular}{lr} 
Groups & Function \\
Leave $<40$ & -11.901 \\
\hline Leave $>39$ & 11.901
\end{tabular}

\section{I DISCUSSION AND CONCLUSION}

Our U.K. analysis is based on a questionnaire carried out on a representative sample of the U.K. population stratified by sociodemographic variables and geographic region (NUTS1). Responses to the questionnaire were subjected to multidimensional analysis-a combination of multiple correspondence analysis and CA.

Findings from our multicountry analysis show how cultural milieus are characterized by five basic, embodied, affectladen, generalized worldviews (defined as "symbolic universes"). Four (ordered universes, interpersonal bond, caring society, niche of belongingness) out of five symbolic universes consist of a way of interpreting reality by reason of a specific anchorage (i.e., ethical norm, emotional experience of interpersonal bond, institutional order, belongingness). One symbolic universe (others' world) can be viewed as the anomic reaction to the failure of those anchorages. The symbolic universes also have been analysed in terms of three basic dimensions of meaning that the multidimensional analysis showed to underpin their reciprocal similarities and differences: (a) the connotation of the experience (friend vs. foe); (b) the form of engagement with reality (active vs. passive); (c) the demand (sense/identity vs. systemic order/functionality). Moreover, the sociodemographic profiles of the symbolic universes have been mapped, in terms of characteristics of people associated with each symbolic universe, as well as the distribution of the symbolic universes across countries.

Focusing on Brexit, by means of a discriminant analysis, we have presented how the incidence of the symbolic universes (and of the underpinning basic dimensions of meaning within the 12 UK macroregions') enables differentiation of the macroregions with a high proportion of "leave" referendum votes from those with a low proportion. More specifically, the high "leave" macroregion are characterized by higher relevance of cultural forms that are related to local identity/belongingness.

Taken as a whole, these findings are consistent with the SCPT framework the study is grounded on. Indeed, they provide empirical evidence for the recognition that symbolic universes matter: The generalized meanings they provide help explain Leave/Remain choice in the Brexit referendum.

It is worth highlighting the methodological and practical implications associated with this theoretical enhancement. From a methodological standpoint, findings reported in this study indicate the capacity of the SCPT cultural analysis to identify relevant aspects of the cultural milieu predictive of Brexit choices within the U.K. regional territories. From a practical standpoint, this result is relevant because it suggests that the knowledge of the incidence of the symbolic universes can be a key facet in the understanding of the electoral dynamics and more generally in people's institutional attitudes and commitment.

Focusing on the specific significance of the findings presented above, one can note that they are consistent with a view of the Brexit vote in terms of an identity enactment (Curtice, 2016; Kenny, 2016), rather than as a reaction to anomy. This interpretation is suggested, in particular, by the role played by the incidence of interpersonal bond and niche of belongingness - that is, the two symbolic universes characterized by the relevance of the identity networkin territorial areas with a high percentage of Leave votes as well as by the fact that the anomic symbolic universe does not play any discriminant role.

Looking at the current political landscape in Europe after the economic crisis, it would appear that the long-standing tension between traditionalists and modernists has found new life. The conditions are ripe for another catalysing contest. The modern sensibility of individual voluntarism and independence has long struggled with growing systemic failures and internal demands for equity, and confidence in its institutions has slowly ebbed. On one side, this tension is addressed through a reaffirming of boundaries and identities, whereas on the other hand, it is answered by an expansion and overcoming of identity boundaries. 


\section{ORCID}

Giuseppe A. Veltri (i) http://orcid.org/0000-0002-9472-2236

\section{REFERENCES}

Arnorsson, A., \& Zoega, G. (2016). On the causes of Brexit. Working Papers in Economics and Finance, 1605, Birkbeck College.

Aronoff, M. J., \& Kubik, J. (2013). Anthropology and political science: A convergent approach. In New York. Oxford: Berghahn Books.

Benzecri, J. P. (1992). Correspondence analysis handbook. New York: Marcel Dekker.

Carli, R., \& Paniccia, R. M. (1999). Psicologia della formazione [Psychology of the training]. Bologna: II Mulino

Carli, R., \& Salvatore, S. (2001). L'immagine della psicologia: Una ricerca sulla popolazione del Lazio [The image of psychology: A study on the Lazio's population]. Roma: Kappa.

Ciavolino, E., Redd, R., Avdi, E., Falcone, F., Fini, V., Kadianaki, I., ... Salvatore, S. (2017). Views of context. An instrument for the analysis of the cultural milieu. A first validation study. Electronic Journal of Applied Statistical Analysis, 10(2), 599-628.

Curtice, J. (2016). Brexit: Behind the referendum. Political Insight, 7(2), 4-7.

Dorling, D. (2016). Brexit: The decision of a divided country. BMJ, i3697.

Goodwin, M. J., \& Heath, O. (2016). The 2016 referendum, Brexit and the left behind: An aggregate-level analysis of the result. The Political Quarterly, 87(3), 323-332.

Henderson, A., Jeffery, C., Liñeira, R., Scully, R., Wincott, D., \& Wyn Jones, R. (2016). England, englishness and brexit. The Political Quarterly, 87(2), 187-199.

Kaufmann, E. (2016). It's NOT the economy, stupid: Brexit as a story of personal values. British Politics and Policy at LSE.

Kenny, M. (2016). The genesis of English nationalism. Political Insight., 7, 8-11.

Landauer, T. K., Foltz, P. W., \& Laham, D. (1998). Introduction to latent semantic analysis. Discourse Processes, 25, 259-284.

Mannarini, T. M., Nitti, M., Ciavolino, E., \& Salvatore, S. (2012). The role of affects in culture-based interventions: Implications for practice. Psychology, 3, 569-577. https://doi.org/10.4236/psych. 2012.38085

Osgood, C. E., Suci, G. J., \& Tannenbaum, P. H. (1957). The measurement of meaning. Urbana: University of Illinois Press.

Recrire consortium (2017). TR syncronic analysis. Deliverable 3.2. Unpublished manuscripts. Retrieved at www.recrire.eu.

Salvatore, S. (2017). The cultural psychology of desire. In J. Valsiner, G. Marsico, N. Chaudhary, T. Sato, \& V. Dazzani (Eds.), Psychology as the science of human being. The Yokohama Manifesto. Annales of Theoretical Psychology (pp. 33-49). Heidelberg, New York, Dordrecht, London: Springer.

Salvatore, S. (2018). Culture as dynamics of sense-making. A semiotic and embodied framework for socio-cultural psychology. In J. Valsiner, \& A. Rosa (Eds.), Cambridge handbook of culture \& psychology.

Salvatore, S., Fini, V., Mannarini, T., Valsiner, J., \& Veltri, G. A. (Eds.) (forthcoming). What future for Europe? Symbolic Universes in the time of (post)crisis. Heidelberg, New York, Dordrecht, London: Springer.

Salvatore, S., Fini, V., Mannarini, T., Veltri, G. A., Avdi, E., Battaglia, F., ... on behalf of the Re.Cri.Re. Consortium. (2018). Symbolic universes between present and future of Europe. First results of the map of European societies' cultural milieu. PLoS One, 13(1), e0189885. https://doi.org/10.1371/journal.pone.0189885

Salvatore, S., Tonti, M., \& Gennaro, A. (2017). How to model sensemaking. A contribution for the development of a methodological framework for the analysis of meaning. In M. Han, \& C. Cunha (Eds.), The subjectified and subjectifying mind (pp. 245-268). Charlotte (NC, USA): Information Age Publishing.

Salvatore, S., \& Venuleo, C. (2013). Field dependency and contingency in the modelling of sensemaking. Papers on Social Representation [on Line Journal], 22(2), 21.1-21.41.

Salvatore, S. (2016). Psychology in black and white. The project of a theory-driven science. Charlotte NC: Information Age Publishing.

Valsiner, J. (2007). Culture in minds and societies. Foundations of cultural psychology. New Delhi: Sage.

Valsiner, J., \& Rosa, A. (Eds.) (2007). The Cambridge handbook of sociocultural psychology. Cambridge: Cambridge University Press.

Venuleo, C., Salvatore, S., \& Mossi, P. G. (2015). The role of cultural factors in pathological gambling. Journal of Gambling Studies, 31(4), 1353-1376. https://doi.org/10.1007/s10899-014-9476-z 


\section{SUPPORTING INFORMATION}

Additional supporting information may be found online in the Supporting Information section at the end of the article.

How to cite this article: Veltri GA, Redd R, Mannarini T, Salvatore S. The identity of Brexit: A cultural psychology analysis. J Community Appl Soc Psychol. 2019;29:18-31. https://doi.org/10.1002/casp.2378 